\title{
Telecom wavelength optical processor for wideband spectral analysis of radiofrequency signals
}

\author{
Anne Louchet-Chauvet, ${ }^{1,2}$ Perrine Berger, ${ }^{3}$ Pascale Nouchi, ${ }^{3}$ Daniel \\ Dolfi, ${ }^{3}$ Alban Ferrier, ${ }^{4,5}$ Philippe Goldner, ${ }^{4}$ and Loïc Morvan ${ }^{3}$ \\ ${ }^{1}$ Laboratoire Aimé Cotton, UMR9188, CNRS, Univ. Paris-Sud, Université Paris-Saclay, 91400 Orsay, France \\ ${ }^{2}$ Institut Langevin, ESPCI Paris, PSL University, CNRS, 1 rue Jussieu, 75005 Paris, France \\ ${ }^{3}$ Thales Research and Technology, Palaiseau, France \\ ${ }^{4}$ Chimie Paris Tech, PSL University, CNRS, Institut de Recherche de Chimie Paris, 75005 Paris, France \\ ${ }^{5}$ Faculté des Sciences et Ingéniérie, Sorbonne Université, UFR933, 75005 Paris, France
}

\begin{abstract}
In this paper we present a spectral analyzer for wideband RF signals with a spectral holeburning-based architecture operating at telecom wavelength. This device is based on a codoped $\mathrm{Er}^{3+}: \mathrm{Sc}^{3+}: \mathrm{Y}_{2} \mathrm{SiO}_{5}$ crystal whose inhomogeneous linewidth allows for wideband operation. With time-resolved holeburning spectroscopy experiments we study the homogeneous linewidth and spectral diffusion in an unusual magnetic field configuration. We finally demonstrate the spectral analysis of RF signals with $28 \mathrm{GHz}$ instantaneous bandwidth and $\mathrm{MHz}$ resolution. This work opens the way towards more complex architectures such as direction finding with wideband capacity.
\end{abstract}

\section{INTRODUCTION}

For decades, analyzing radiofrequency signals over a wide bandwidth in real time has been identified as a significant technological challenge. This challenge is amplified with the proliferation of transmitters for radar and communication systems operating over increasingly broad regions of the electromagnetic spectrum. Because they present broad bandwidth capabilities more naturally than electronics, optical solutions are under active development for RF signal processing.

In this context, a spectrum analyzer architecture based on spectral hole burning, capable of outcompeting digital spectrum analyzers over many aspects was proposed [1]. It is based on the spectral hole burning (SHB) mechanism that occurs for example in cryogenically-cooled rare-earth ion-doped crystals. The principle of the spectral analyzer consists in exposing the SHB material to the optically carried RF signal under investigation and periodically reading out the resulting spectral photograph with a monochromatic laser scanned over the altered absorption profile. Thus, by just measuring the SHB material transmission previously illuminated by an optically carried RF signal, one can achieve a spectral analyzer with buffer memory. The bandwidth and the resolution of such a device are linked to the inhomogeneous and homogeneous widths of the SHB medium, respectively. It is now being produced as a device by S2Corporation [2] and operates at $793 \mathrm{~nm}$. This wavelength is imposed by the choice of the rare earth ion-doped crystal (Tm:LuYAG) allowing a $35 \mathrm{GHz}$ instantaneous bandwidth together with a sub-MHz resolution.

Transposing such an architecture at telecom wavelength would be beneficial. Indeed, various optical components have been developed at $1.5 \mu \mathrm{m}$ with better performances than at $800 \mathrm{~nm}$. One can find for example reliable and powerful laser sources, optical amplifiers with lower non-linearities, photodetectors combining high saturation level, large bandwidth and high linearity. In addition, other functionalities like optical distribution could share the same electro-optical conversion. However, switching to telecom wavelength requires the use of erbium-doped crystals.

In the quest for high spectral resolution, Er-doped $\mathrm{Y}_{2} \mathrm{SiO}_{5}$ (also known as YSO) is known to be one of the best candidates [3]. Nevertheless, it also has the most narrow inhomogeneous broadening $(0.4-1 \mathrm{GHz})$ compared to other erbium-doped crystals [4] (up to $300 \mathrm{GHz}$ for $\mathrm{Er}: \mathrm{LiNbO}_{3}$ ), limiting the bandwidth of signal processing architectures realized in this crystal [5].

In rare earth ion-doped crystals the inhomogeneous broadening is given by the chemical or structural defects created during the growth of the crystal. Replacing a significant fraction of the host matrix elements by a different species with a significantly different ionic radius tends to increase the disorder due to local deformation of the lattice and can lead to an enhancement of the static inhomogeneous broadening. The nature of the additional dopant determines whether it will also induce new decoherence channels, reduce existing ones or enhance them for example via energy transfer [6].

The inhomogeneous broadening enhancement has been successfully observed in Er:YSO with the addition of a few percent of $\mathrm{Eu}^{3+}[7]$ or $\mathrm{Sc}^{3+}$ ions [8]. With europium codoping it increases by factor of $11 \mathrm{GHz} / \% \mathrm{Eu}$. A proofof-principle direction finding demonstration was reported in [9] at $1.5 \mu \mathrm{m}$ using a Er:Eu:YSO crystal, with a $5 \mathrm{GHz}$ bandwidth, ultimately limited by the $14 \mathrm{GHz}$ inhomogeneous width of this crystal. Codoping with $1 \%$ scandium leads to a $25 \mathrm{GHz}$ inhomogeneous broadening, probably due to its larger discrepancy in ionic radius compared with $\mathrm{Y}^{3+}$ ions [8]. We focus on this crystal in order to reach a processing bandwidth exceeding $20 \mathrm{GHz}$.

In this paper we address the spectral analysis of wideband $\mathrm{RF}$ signals at telecom wavelength in a $0.003 \%$ Er: $1 \%$ Sc:YSO crystal. In the first section, we justify the choice of the applied magnetic field, and present original spectroscopic results highlighting the spectral 
and temporal resolution limits offered by this crystal under such a magnetic field. In the second section, we present the spectral analysis setup and the experimental results. We focus on the processing bandwidth, spectral resolution, and single-shot dynamic range. Finally, in the last section, we discuss some lines of improvement regarding the dynamic range, spectral resolution, and low temperature operation.

\section{SPECTROSCOPIC STUDY OF ER:SC:YSO}

\section{A. Choice of magnetic field}

Trivalent erbium ions in YSO possess one of the largest electronic spins among all chemical elements. As a consequence, in an ensemble, erbium ions tend to interact strongly, leading to decoherence. This decoherence mainly originates from local magnetic field fluctuations due to the surrounding erbium spin fluctuations. To minimize this decoherence, the standard strategy consists in keeping the doping concentration low and applying a magnetic field of more than $1 \mathrm{~T}$ [10]. In this regime, the spin dynamics is governed by the interaction of the spins with the phonon bath, meaning that any increase in temperature severely intensifies the decoherence. Anticipating the future necessity to transpose our experiment to a more mature technology level, the robustness to temperatures up to $4 \mathrm{~K}$ is compulsory in order to be compatible with standard closed-cycle cryocoolers. Therefore we rule out the strong magnetic field configuration, at the cost of a moderate spectral resolution.

For intermediate values of the magnetic field (between $1 \mathrm{mT}$ and $1 \mathrm{~T}$ ), the Kramers doublet are split with a typical sensitivity of 30 to $200 \mathrm{GHz} / \mathrm{T}$ [11], with population lifetimes than may exceed that of the excited states [12]. This gives rise to unwanted, persistent symmetric spectral features around each spectral hole (side holes and antiholes) that are not relevant for spectral analysis.

Therefore we aim for small magnetic fields below $1 \mathrm{mT}$. In such a low field regime, the interaction of the erbium spins with the phonon bath becomes negligible and their dynamics is dominated by cross-relaxation between close erbium spins, often referred to as flip-flops. This mechanism is the main limit to the homogeneous linewidth [13] and is expected to be rather immune to temperature increase. Besides, it was shown in a previous study that the presence of $1 \% \mathrm{Sc}$ ions leads to a tenfold increase in the $\mathrm{Er}^{3+}$ electron spin linewidth in the ground state, which in turn slows down the flip-flop processes [8]. The question remains whether the homogeneous linewidth in such a low field configuration is small enough to allow spectral analysis with sub-MHz spectral resolution. We address this question in the following.
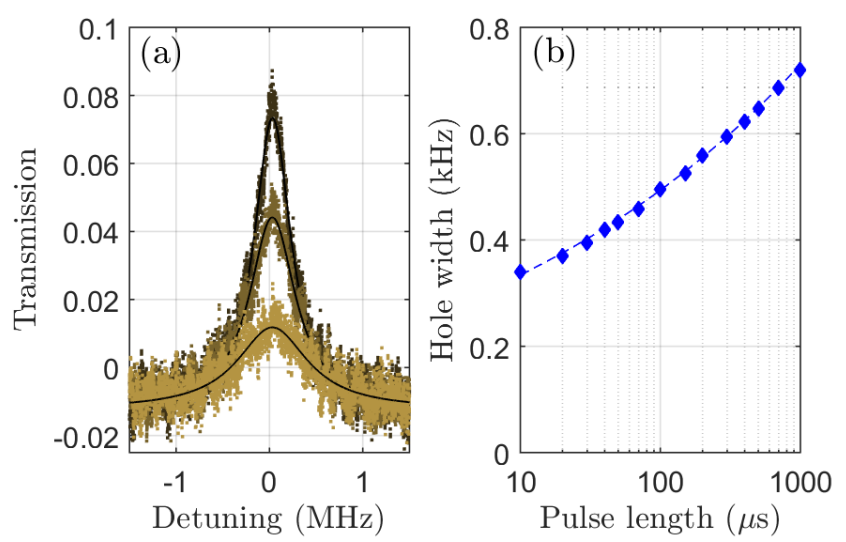

FIG. 1. (a) Example of hole shape at $T=3.8 \mathrm{~K}$ for three particular values of $T_{W}(110 \mu \mathrm{s}, 1.1 \mathrm{~ms}$ and $10.1 \mathrm{~ms})$. Solid lines are lorentzian fits to the data. (b) Evolution of hole width with the burning pulse duration indicating power broadening, with $T_{W}=50 \mu \mathrm{s}$. Note the logarithmic timescale.

\section{B. Time-resolved hole-burning spectroscopy}

We perform a spectroscopic study to determine the spectral resolution limit of the Er:Sc:YSO crystal at very low magnetic field. To that end, we use a distributedfeedback fiber laser (Koheras) with a linewidth below $3 \mathrm{kHz}$, tuned to the ${ }^{4} I_{15 / 2}(1) \leftrightarrow{ }^{4} I_{13 / 2}(1)$ transition at $1536 \mathrm{~nm}$. The laser power on the crystal is around $2 \mathrm{~mW}$ and the beam waist is $1 \mathrm{~mm}$. The burning is achieved with a single rectangular $20 \mu$ s pulse, while the probing is made of a rectangular $200 \mu$ s pulse, linearly chirped over $10 \mathrm{MHz}$, emitted after the burning pulse with a delay $T_{W}$. The chirp is performed thanks to a Gooch \& Housego fibered acousto-optic modulator driven with a arbitrary waveform generator Tektronix AWG5004. The transmission spectra are collected on a Newfocus 1811 photodiode and are digitized thanks to an Agilent DSO5034A oscilloscope. The spectra are post-processed via a chirpcompensation algorithm [14].

We cool the crystal down below $4 \mathrm{~K}$ in the gas phase of an ICE Oxford helium cryostat. No magnetic field is applied but a $102 \mu \mathrm{T}$ field is measured at the crystal location due to remanent magnetization of the optical table and cryostat, directed mainly towards the ground. The 50 -fold enhancement of the inhomogeneous linewidth due to the presence of scandium leads to a very low absorption coefficient $(0.14 / \mathrm{cm}$ at the center of the line). To reach a significant optical depth we fold the beam so that it goes through the 9-mm-long sample three times. This way we reach $\alpha L=0.4$.

We observe a spectral hole with up to $10 \%$ excess transmission that we fit with a lorentzian function as illustrated in Fig. 1 (a). At short delay $(100 \mu \mathrm{s})$ the hole FWHM is around $500 \mathrm{kHz}$. By varying the waiting time $T_{W}$ between burning and probing up to $10 \mathrm{~ms}$, we observe a progressive broadening of the spectral hole (see Fig. 2). 


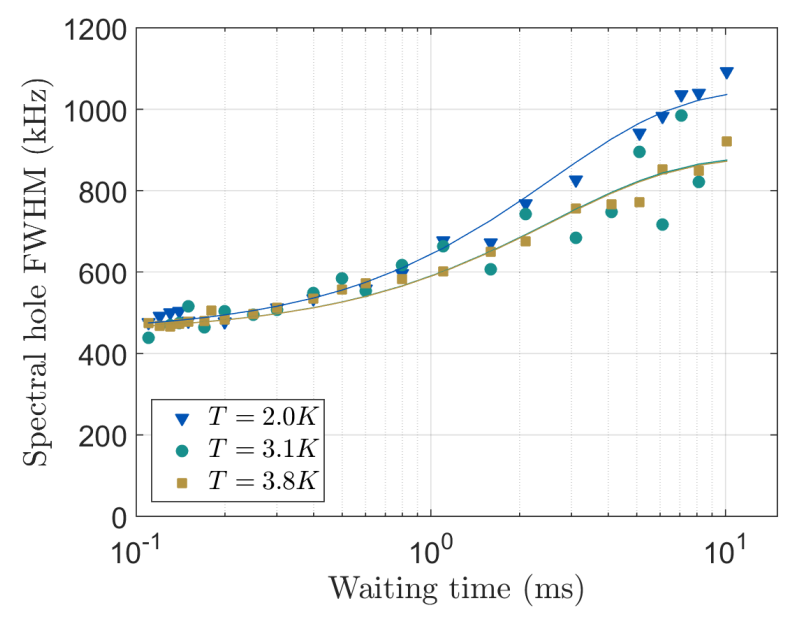

FIG. 2. Evolution of the width (FWHM) of a spectral hole. Solid lines are fits to the data following equation 1 . The data is quieter at $2 \mathrm{~K}$ thanks to helium superfluid behaviour below the helium Lambda point $(2.17 \mathrm{~K})$. Above that point, strong turbulences occur.

The total width reaches between 0.9 and $1.1 \mathrm{MHz}$ depending on the temperature. This broadening is usually described via the following equation [10],

$$
\Gamma_{\text {hole }}\left(T_{W}\right)=2 \Gamma_{0}+\Gamma_{\mathrm{SD}}\left(1-e^{-R T_{W}}\right),
$$

where $R$ is the rate of spin flips in the ions' environment, $\Gamma_{0}$ is the linewidth at zero delay (including the natural homogeneous linewidth $\gamma_{h}$, the laser linewidth, power broadening, and instantaneous spectral diffusion), and $\Gamma_{\mathrm{SD}}$ is the maximum broadening due to spectral diffusion. The factor of 2 stems from the double interrogation of the atoms during burning and probing. From other spectroscopy measurements performed in Er:YSO [10] under a larger magnetic field we expect $\Gamma_{S D}>200 \mathrm{kHz}$ and $R<1 \mathrm{kHz}$. We use Eq. 1 to fit the data in Fig. 2 and obtain $\Gamma_{0}=225 \mathrm{kHz}, \Gamma_{S D}=430$ to $600 \mathrm{kHz}$ depending on the sample temperature and $R=390 \mathrm{~Hz}$.

To assess the contribution of power-related broadening (i.e. power broadening and instantaneous spectral diffusion) to the hole width, we explore various durations for the burning pulse from $10 \mu$ s to $1 \mathrm{~ms}$ and observe that the spectral holes broadens as the pulse length is increased [see Fig. 1(b)]. For $10 \mu$ s burning pulse the spectral hole is as narrow as $350 \mathrm{kHz}$. Therefore we infer that the homogeneous linewidth $\gamma_{h}$ is at most $175 \mathrm{kHz}$.

As the waiting time increases, the atomic populations stored in shelving states decay, progressively filling the hole. In Fig. 3 we plot the evolution of the hole area with the waiting time and observe an exponential decay with characteristic time $5 \mathrm{~ms}$ towards a non-zero background value. We attribute this decay to the population relaxation from the excited state, and the constant background to long-term storage in the Zeeman sublevels. The excited state lifetime does not depend on the temperature and is similar to the ones measured in Er:YSO

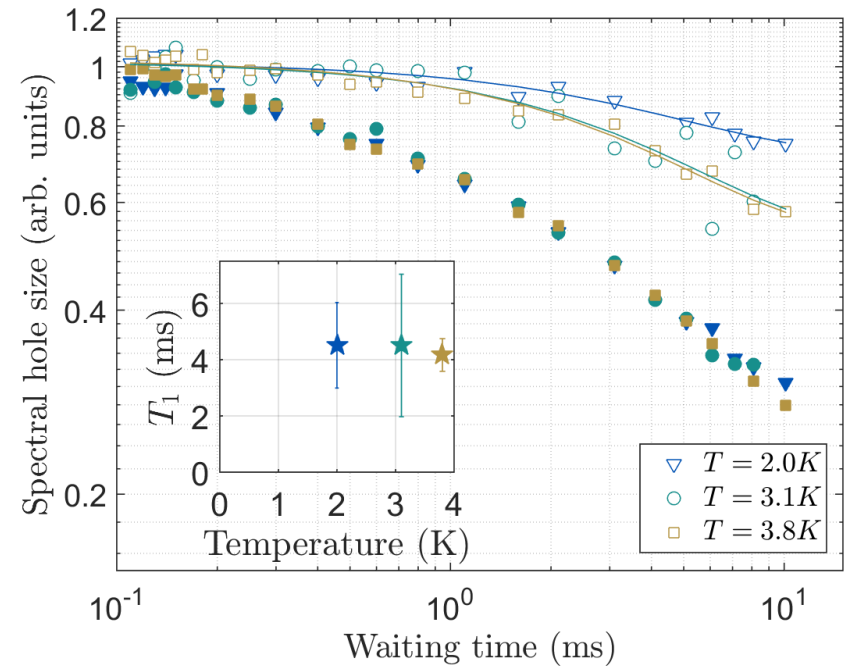

FIG. 3. Spectral hole decay, leading to the spectral analyzer's temporal resolution. The open and closed symbols respectively correspond to the hole area and the hole height. The spectral hole height decays faster than the hole area due to the spectral diffusion-induced broadening.

(10 ms) and Er:Eu:YSO (8 ms) [15]. The constant background, on the other hand, seems to decrease as the sample warms up, consistent with a shortening of the Zeeman sublevel population lifetime leading to less accumulation.

We can estimate the temporal resolution of a spectral analyzer based on our Er:Sc:YSO crystal by plotting the spectral hole height as a function of the readout delay (see Fig. 3). The hole height decreases by a factor of 3 within $10 \mathrm{~ms}$. The persistent part due to partial storage in the electronic Zeeman levels will result as a remanence effect in the spectral analyzer, but with a limited impact thanks to spectral diffusion.

From this rather simple spectroscopic study we derive two fundamental limitations for the spectral analyzer based on such a crystal: its spectral resolution will be at best $0.5 \mathrm{MHz}$, and its temporal resolution around $5 \mathrm{~ms}$.

\section{SPECTRAL ANALYSIS}

In this section we present an ensemble of experiments demonstrating spectral analysis in Er:Sc:YSO. After describing the experimental setup, we address its spectral bandwidth and resolution capacity, then we measure its dynamic range.

\section{A. Description of the setup}

Ideally, the spectral analyzer operates in two steps: a writing step, where a monochromatic laser is modulated by the RF signal with the help of a Mach-Zehnder 


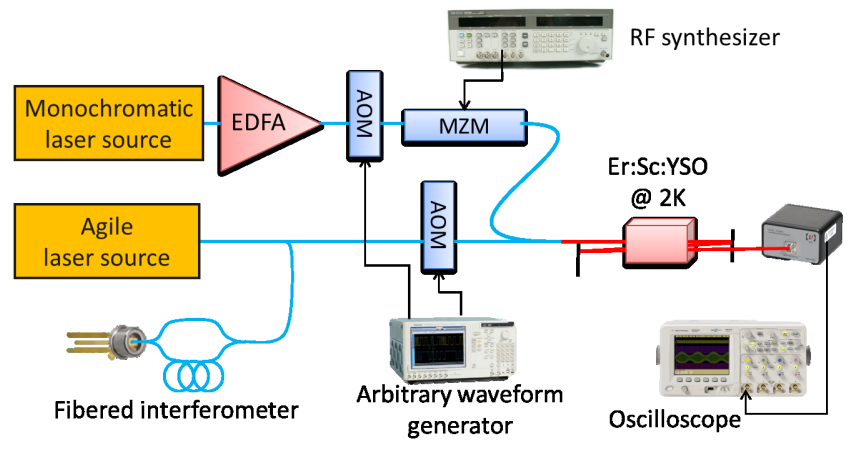

FIG. 4. Experimental setup. The optical setup is mostly fibered, except in the vicinity of the cryostat. EDFA: erbium-doped fiber amplifier; MZM: Mach-Zehnder modulator; AOM: acousto-optic modulator.

modulator and burns spectral holes in the crystal absorption profile, and a probing step, where a second laser is periodically chirped and probes the modified absorption profile. In the context of efficient electromagnetic spectrum surveillance, a 100\% probability of intercept (POI) is necessary, which is possible only if the writing step and the probing step are simultaneous. In that case the corresponding laser beams must be efficiently isolated from each other (by means of a non-colinear arrangement for example) while shining a common volume of the SHB medium. In the present work, due to the low sample absorption, we use a multipass configuration to enhance the contrast of the spectral holes and thus the dynamic range of the device (see Fig. 4). Therefore we combine the programming and the probing beam into a single mode fiber to ensure perfect spatial overlap, and temporally alternate the writing and the probing steps with the help of acousto-optic modulators acting as gates, leading to a $50 \%$ POI. The alternation of the writing and probing step is achieved with a $2 \mathrm{~ms}$ period.

The source used for the writing step is the Koheras laser described in Sec. II, amplified with a Manlight erbium-doped fiber amplifier. The Mach-Zehnder modulator (MZM) is a iXblue Photonics fibered lithium niobate modulator with a $25 \mathrm{GHz}$ modulation bandwidth, operating close to the quadrature point.

The agile laser source used for the probing step is a distributed feedback laser (Gooch \& Housego AA1401), rapidly tunable over $30 \mathrm{GHz}$. The instantaneous laser frequency is controlled with a sensitivity of $15 \mathrm{GHz} / \mathrm{V}$ with the help of an arbitrary function generator (Tektronix AFG3021B) generating slightly smoothed triangle waves at $500 \mathrm{~Hz}$. The frequency excursion is quite reproducible, which is why we choose not to setup a feedback loop. The exact frequency excursion is measured a posteriori with a fibered unbalanced interferometer (with a $100 \mathrm{MHz}$ free spectral range) coupled into a Hamamatsu G8338 detector. The useful part of the chirp covers around $28.3 \mathrm{GHz}$. The Koheras laser is detuned by $8 \mathrm{GHz}$ from the DFB mean frequency so as to analyze $\mathrm{RF}$ frequencies from -22

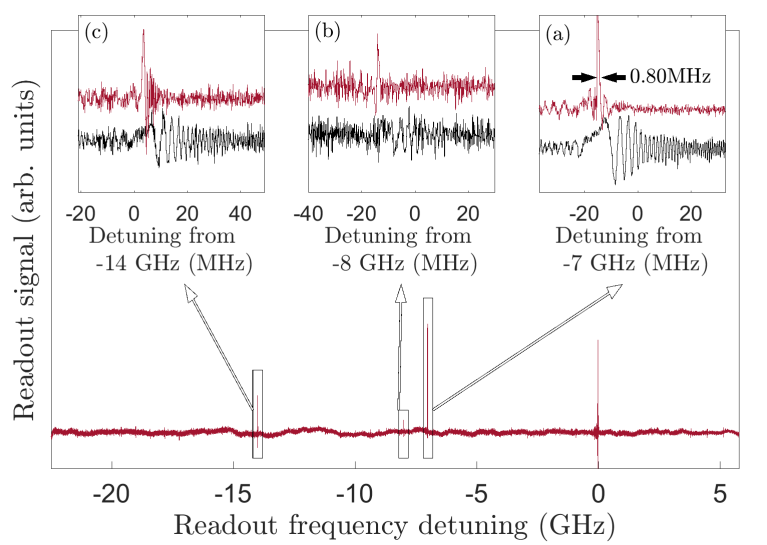

FIG. 5. Simultaneous detection of several frequencies demonstrating instantaneous wideband capacity. The main graph shows a typical readout signal corrected from ringdown artefacts, given the RF source is set to $7 \mathrm{GHz}$. Insets (a), (b) and (c) show closeups of the readout signal (in red) at -7 , -8 and $-14 \mathrm{GHz}$, respectively, together with the raw signal exhibiting strong ringdown effects (in black).

\section{to $6 \mathrm{GHz}$.}

Due to the fast frequency excursion (30 GHz in $1 \mathrm{~ms})$, ringdown signatures are expected to occur when probing spectral features narrower than $6 \mathrm{MHz}$. A correction algorithm is achieved in a post-processing step [14], assuming a constant chirp rate over the whole chirp interval. In practice the chirp rate varies by around $13 \%$ (between 28.7 and $32.7 \mathrm{MHz} / \mu \mathrm{s}$ ) during the chirp (but is reproducible to better than 1\%) and this can lead to an imperfect compensation of the ringdown effect and some inaccuracy in the RF frequency values measured by our spectral analyzer.

\section{B. Spectral bandwidth and resolution}

We successively generate monochromatic RF signals ranging from $22 \mathrm{GHz}$ to $1 \mathrm{GHz}$ with the help of a synthesizer HP 83731A. This synthesizer is able to deliver between 14 and $23 \mathrm{dBm}$ maximum output power depending on the RF frequency.

Figure 5 shows the readout signal when the synthesizer is set to $7 \mathrm{GHz}$, after application of the correction algorithm. Several features are visible, at $0,-7,-8$ and $-14 \mathrm{GHz}$, respectively: the $-7 \mathrm{GHz}$ and $-14 \mathrm{GHz}$ contributions are due to the RF synthesizer generating a $7 \mathrm{GHz}$ signal with additional second harmonics due to nonlinearities in the synthesizer. The $-8 \mathrm{GHz}$ component corresponds to the RF signal recorded by the spectral analyzer when the RF synthesizer was set to $8 \mathrm{GHz}$ a few seconds earlier, indicating a long-term remanence of the device, compatible with the long-lived partial population storage in the crystal already evidenced in Fig. 3. This remanence degrades the temporal resolution capac- 


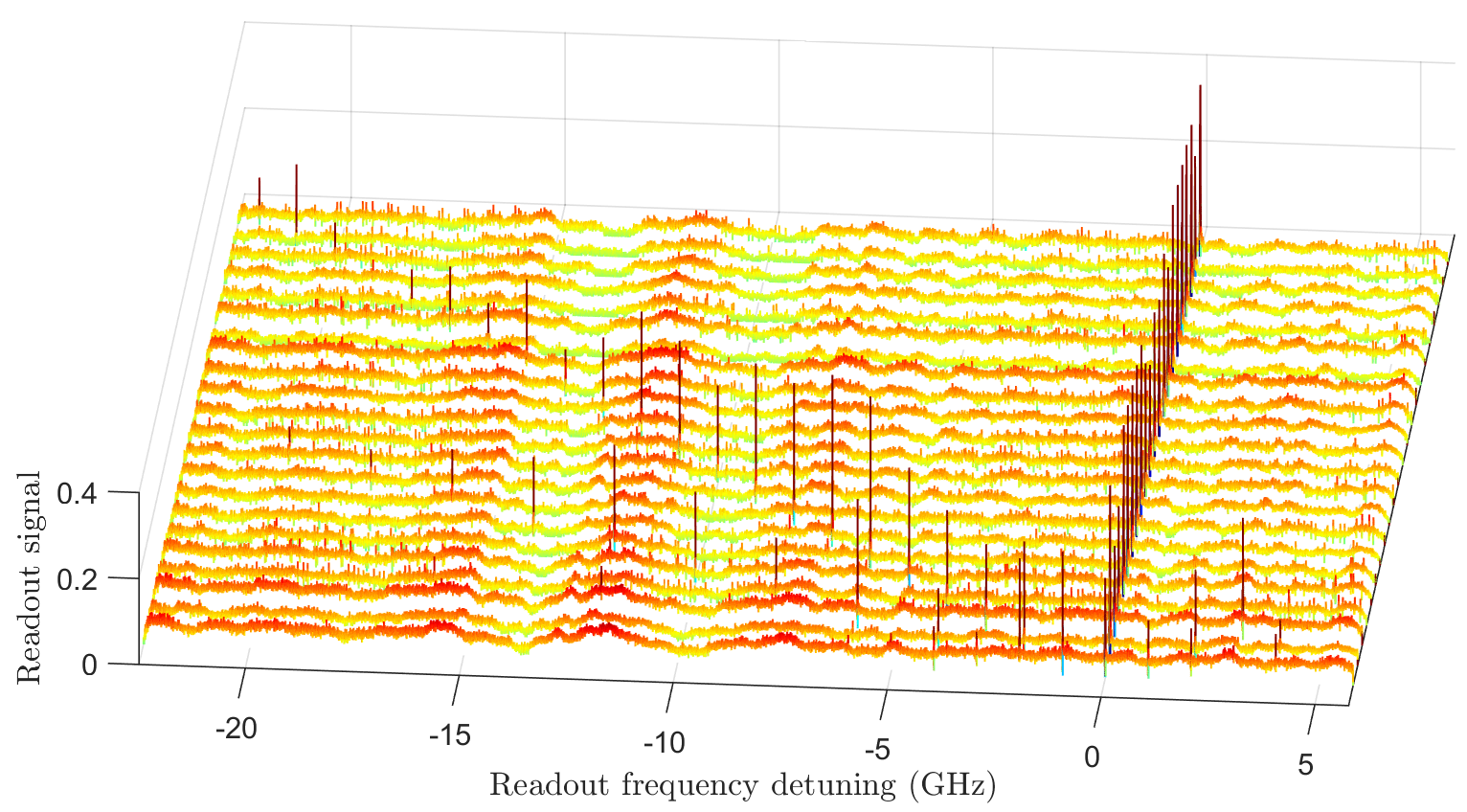

FIG. 6. Demonstration of wideband operation. The RF synthesizer successively generates a monochromatic RF signal at frequencies from 22 to $1 \mathrm{GHz}$ by steps of $1 \mathrm{GHz}$. Each line corresponds to a readout signal when the synthesizer is set to a given $\mathrm{RF}$ frequency.

ity of the spectrum analyzer. This could be avoided by applying an appropriate magnetic field so that the Zeeman levels are either degenerate, or short-lived. Finally, the AOM gating does not fully cancel the burning laser optical power during the readout step, which gives rise to a beatnote between the two lasers. This beatnote is filtered out by the photodetector cutoff around $110 \mathrm{MHz}$ but still visible around $0 \mathrm{GHz}$. The accuracy of the peak positions is of the order of $\pm 20 \mathrm{MHz}$ due to the chirp rate variations that are not taken into account in the ringdown correction algorithm as mentioned in Sec. III A.

We now plot in Fig. 6 all the readout signals as the synthesizer is manually stepped down in frequency from 22 to $1 \mathrm{GHz}$. Similarly to Fig. 5, both first and second order harmonics are detected when in the analyzer $28.3 \mathrm{GHz}$ spectral range. Therefore our device is able to detect several simultaneous frequencies in a single readout over the whole $28 \mathrm{GHz}$ range. This illustrates the instantaneous wideband analysis capacity of our device.

Once the ringdown effects are corrected we measure a typical peak width (FWHM) of $0.8 \mathrm{MHz}$ [see Fig. 5(a)]. Comparing this value with the results shown in Fig. 2, we infer that the analyzer spectral resolution is limited by the homogeneous linewidth of our crystal and the spectral diffusion process.

We confirm this resolution by performing the spectral analysis of a phase-modulated $1 \mathrm{GHz}$ signal with modulation frequency comprised between 0.8 and $3 \mathrm{MHz}$ (see Figure 7). Again, this signal is generated by the HP 83731A synthesizer. The sidebands are visible down

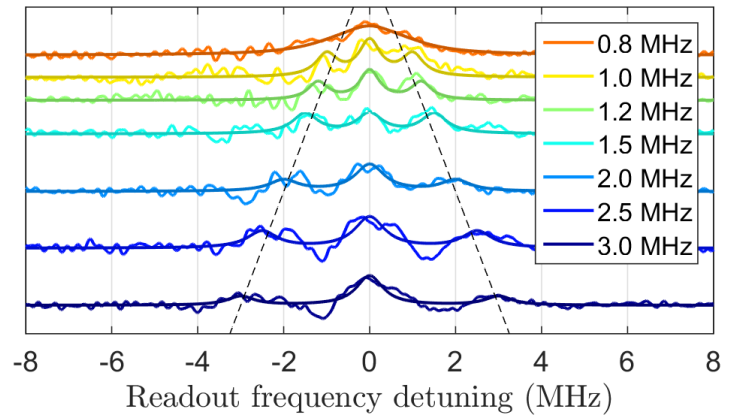

FIG. 7. Spectral analysis of a phase-modulated signal centered at $1 \mathrm{GHz}$. The modulation frequency is varied between 0.8 and $3 \mathrm{MHz}$ to assess the frequency resolution. The dashed line is a guide to the eye indicating the expected position of the sidebands.

to $1 \mathrm{MHz}$ modulation frequency. Given the total detection bandwidth $(28.3 \mathrm{GHz})$, we demonstrate spectral analysis with around 28000 spectral channels. With our refresh rate $(500 \mathrm{~Hz})$, our system performs 14 million frequency measurements per second.

\section{Dynamic range}

We investigate the single-shot dynamic range of our setup by successively achieving the spectral analysis of a continuous monochromatic RF signal at 1 or $3 \mathrm{GHz}$ 


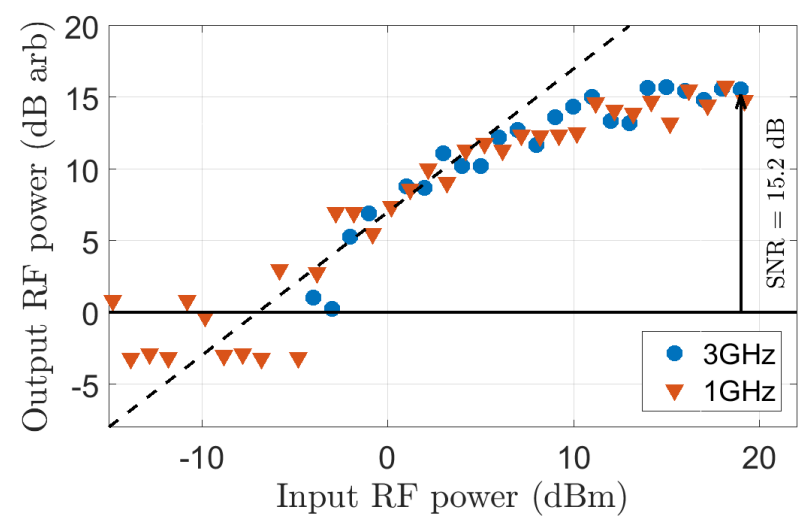

FIG. 8. Single-shot dynamic range of the Er:Sc:YSO-based spectrum analyzer for monochromatic RF signals at 1 and $3 \mathrm{GHz}$. The solid line materializes the noise background, whereas the dashed line is a guide to the eye highlighting the linear part of the input-output power dependence.

and varying the $R F$ power from -13 to $+19 \mathrm{dBm}$ nominal power. The corresponding peak heights are plotted in Fig. 8. As expected the readout peak power linearly follows the input power before saturating above $10 \mathrm{dBm}$ input power. From this study we estimate the dynamic range of our spectral analyzer $15.2 \mathrm{~dB}$, which is low compared to state-of-the-art wideband spectrum analyzers [2], but encouraging given the low absorption of the sample (around $\alpha L \simeq 0.4$ with the triple pass configuration, to be compared with $\alpha L=1-3$ in the S2corp device [16]).

\section{DISCUSSION}

\section{A. Improving the dynamic range}

In this work, the low absorption of our crystal is clearly a limit to the sensitivity and dynamic range of the spectral analyzer, but also to the probability of intercept. Increasing the crystal absorption can be done by increasing the erbium doping rate. This would directly improve the single-shot dynamic range. A high enough absorption would also help drop the multipass beam configuration and perform simultaneous writing and probing, reaching $100 \%$ POI [1]. However, a larger erbium concentration would also enhance the erbium-erbium flip-flop processes [13], leading to a stronger spectral diffusion process and a degradation of the spectral resolution.

\section{B. Going cryogen-free}

The use of closed-cycle cryocoolers in a more technologically mature setup will lead to higher sample temperatures, typically $4 \mathrm{~K}$. We have shown in the spectroscopic study presented in this paper that the spectral diffusion and population decay processes are quite independent on the sample temperature up to 3.8K (see Figs. 2 and 3). This is made possible by the appropriate magnetic field value chosen in this demonstration. Therefore we expect the setup to be transposable to $4 \mathrm{~K}$ with no impact on the spectral resolution and hole lifetime.

\section{Reaching higher spectral resolution}

We have shown that the resolution capacity of our spectrum analyzer $(1 \mathrm{MHz})$ is limited by the homogeneous linewidth and the spectral diffusion process in Er:Sc:YSO. If necessary, a higher resolution should be accessible by applying an external magnetic field. This magnetic field must be strong enough so there are no long-lived structures in the absorption spectrum since this would add unwanted remanence and kill the temporal resolution. The eventual side structures (sides holes and antiholes) are not an issue since they can be removed by performing a post-processing deconvolution of the spectrum.

This magnetic field configuration will however lead to new difficulties, since the decoherence will be dominated by the phonons, making it more difficult to operate at higher temperatures.

\section{CONCLUSION}

In this work, we have implemented a wideband radiofrequency spectral analyzer in a codoped $\mathrm{Er}^{3+}: \mathrm{Sc}^{3+}: \mathrm{Y}_{2} \mathrm{SiO}_{5}$ crystal operating at telecom wavelength and reached an unprecedented $28 \mathrm{GHz}$ instantaneous bandwidth. The spectral resolution is around $1 \mathrm{MHz}$, compatible with radar spectrum analysis. The modest single-shot dynamic range $(15 \mathrm{~dB})$ is due to the limited absorption coefficient and size of the crystal. We have included spectroscopic studies to confirm that the spectral resolution is limited by the spectral diffusion process in the crystal and that it is not affected by a temperature rise up to $4 \mathrm{~K}$.

\section{ACKNOWLEDGMENTS}

This work was funded by the Direction Générale de l'Armement, and by the Agence Nationale de la Recherche via the DISCRYS project (ANR-14-CE260037-02). This work has been realized in the framework of the joint research laboratory between Laboratoire Aimé Cotton and Thales Research \& Technology. The authors are grateful to Jean-Louis Le Gouët for careful reading of the manuscript. 
[1] M. Colice, F. Schlottau, and K. H. Wagner, Applied optics 45, 6393 (2006).

[2] K. Merkel, S. Bekker, A. Traxinger, C. Stiffler, A. Woidtke, M. Chase, W. Babbitt, C. Harrington, and Z. Barber, in Proc. International Photonics Conference (2016).

[3] T. Böttger, C. W. Thiel, R. L. Cone, and Y. Sun, Phys. Rev. B 79, 115104 (2009).

[4] Y. C. Sun, Rare earth materials in optical storage and data processing applications, Vol. 83 (Liu, G. and Jacquier, B., Springer Berlin Heidelberg, 2005) Chap. 7, p. 379.

[5] V. Crozatier, G. Gorju, J.-L. Le Gouët, F. Bretenaker, and I. Lorgeré, Opt. Lett. 31, 3264 (2006).

[6] M. J. Weber, Phys. Rev. B 4, 2932 (1971).

[7] T. Böttger, C. W. Thiel, R. L. Cone, and Y. Sun, Phys. Rev. B 77, 155125 (2008).

[8] S. Welinski, C. W. Thiel, J. Dajczgewand, A. Ferrier, R. L. Cone, R. M. Macfarlane, T. Chanelière, A. LouchetChauvet, and P. Goldner, Opt. Mater. 63, 69 (2017).

[9] W. R. Babbitt, Z. W. Barber, S. H. Bekker, M. D. Chase, C. Harrington, K. D. Merkel, R. Krishna Mo- han, T. Sharpe, C. R. Stiffler, A. S. Traxinger, and A. J. Woidtke, Laser Physics 24, 094002 (2014).

[10] T. Böttger, C. W. Thiel, Y. Sun, and R. L. Cone, Phys. Rev. B 73, 75101 (2006).

[11] Y. Sun, T. Böttger, C. Thiel, and R. Cone, Phys. Rev. B 77, 085124 (2008).

[12] S. R. Hastings-Simon, B. Lauritzen, M. U. Staudt, J. L. M. van Mechelen, C. Simon, H. de Riedmatten, M. Afzelius, and N. Gisin, Phys. Rev. B 78, 085410 (2008).

[13] B. Car, L. Veissier, A. Louchet-Chauvet, J.-L. Le Gouët, and T. Chanelière, Phys. Rev. B 100, 165107 (2019).

[14] T. Chang, M. Tian, R. K. Mohan, C. Renner, K. D. Merkel, and W. R. Babbitt, Opt. Lett. 30, 1129 (2005).

[15] C. W. Thiel, W. R. Babbitt, and R. L. Cone, Phys. Rev. B 85, 174302 (2012).

[16] K. D. Merkel, C. R. Stiffler, A. Woidtke, A. Traxinger, R. W. Equall, Z. Barber, C. Harrington, R. K. Mohan, C. W. Thiel, R. Cone, et al., "Techniques for spatial spectral holography," (2014), US Patent 8,829,471. 